\title{
AzURITE: An algebraic geometry based package for finding bases of loop integrals
}

\author{
Alessandro Georgoudis ${ }^{\mathrm{a}, \mathrm{c}}$, Kasper J. Larsen ${ }^{\mathrm{b}, \mathrm{c}}$, Yang Zhang, ${ }^{\mathrm{c}, *}$ \\ ${ }^{a}$ Department of Physics and Astronomy, Uppsala University, SE-75108 Uppsala, Sweden \\ ${ }^{b}$ School of Physics and Astronomy, University of Southampton, Highfield, Southampton, \\ SO17 1BJ, United Kingdom \\ ${ }^{c}$ ETH Zürich, Wolfang-Pauli-Strasse 27, 8093 Zürich, Switzerland
}

\begin{abstract}
For any given Feynman graph, the set of integrals with all possible powers of the propagators spans a vector space of finite dimension. We introduce the package AzURITE (A ZURich-bred method for finding master InTEgrals), which efficiently finds a basis of this vector space. It constructs the needed integration-by-parts (IBP) identities on a set of generalized-unitarity cuts. It is based on syzygy computations and analyses of the symmetries of the involved Feynman diagrams and is powered by the computer algebra systems Singular and Mathematica. It can moreover analytically calculate the part of the IBP identities that is supported on the cuts.
\end{abstract}

Keywords: Feynman diagrams, computational algebraic geometry, integration-by-parts identities

\section{PROGRAM SUMMARY}

Program Title: AzURITE

Licensing provisions: GNU General Public License (GPL)

Programming language: Wolfram Mathematica version 10.0 or higher

Supplementary material: A manual in the form of a Mathematica notebook

Nature of problem: Determination of a basis of the space of loop integrals spanned by a given Feynman diagram and all of its subdiagrams

Solution method: Mathematica implementation

\footnotetext{
${ }^{*}$ Corresponding author. E-mail address: Yang. Zhang@phys.ethz.ch

Email addresses: Alessandro.Georgoudis@physics.uu.se (Alessandro Georgoudis), Kasper.Larsen@soton.ac.uk (Kasper J. Larsen)
} 


\section{Introduction}

Precision calculations of the cross sections of Standard Model processes at the Large Hadron Collider (LHC) are crucial to gain a quantitative understanding of the background and in turn improve the ability to extract signals of new physics. This typically requires computations at next-to-next-to leading order (NNLO) in fixed-order perturbation theory, in order to match the experimental precision and the parton distribution function uncertainties. Calculations at this order are challenging because of the large number of contributing Feynman diagrams, which involve loop integrals with high powers of loop momenta in the numerator of the integrand.

A key tool in these calculations are integration-by-parts (IBP) identities [1, 2]. These are relations that arise from the vanishing integration of total derivatives. Schematically, they take the form,

$$
\int \prod_{j=1}^{L}\left(\frac{\mathrm{d}^{D} l_{j}}{i \pi^{D / 2}}\right) \sum_{i=1}^{L} \frac{\partial}{\partial l_{i}^{\mu}} \frac{v_{i}^{\mu}}{D_{1}^{a_{1}} \cdots D_{k}^{a_{k}}}=0,
$$

where the vectors $v_{i}^{\mu}$ are polynomials in the internal and external momenta, the $D_{k}$ denote inverse propagators, and $a_{i} \geq 1$ are integers. In practice, the IBP identities generate a large set of linear relations between loop integrals, and allow a significant fraction of them to be expressed in terms of a finite linear basis. (The fact that the basis of integrals is always finite was proven in ref. [3].) The latter step of solving the linear systems arising from eq. (1) may be carried out by Gauss-Jordan elimination in the form of the Laporta algorithm [4, 5], leading in general to relations involving integrals with squared propagators. There are several implementations of automated IBP reduction publically available: AIR [6], FIRE [7, 8], Reduze [9, 10], LiteRed [11], along with private implementations. Finite field techniques can be used to speed up the computation [12, 13, 14, 15].

A formalism for deriving IBP reductions that do not involve integrals with squared propagators was developed in ref. [16], based on syzygy computations. As observed in ref. [17], the syzygies can be computed with linear algebra methods.

In addition to reducing the contributing Feynman diagrams to a small set of basis integrals, the IBP reductions provide a way to compute these integrals themselves through differential equations [18, 19, 20, 21, 22, 23]. Letting $x_{m}$ denote a kinematical variable, $\epsilon=\frac{4-D}{2}$ the dimensional regulator, and $\mathcal{I}(\mathbf{x}, \epsilon)=\left\{I_{1}(\mathbf{x}, \epsilon), \ldots, I_{N}(\mathbf{x}, \epsilon)\right\}$ the basis of integrals, the result of 
differentiating any basis integral wrt. $x_{m}$ can again be written as a linear combination of the basis integrals by using, in practice, the IBP reductions. As a result, one has a linear system of differential equations,

$$
\frac{\partial}{\partial x_{m}} \mathcal{I}(\mathbf{x}, \epsilon)=A_{m}(\mathbf{x}, \epsilon) \mathcal{I}(\mathbf{x}, \epsilon)
$$

which, supplied with appropriate boundary conditions, can be solved to yield expressions for the basis integrals. This has proven to be a powerful tool for computing two- and higher-loop integrals. As observed in ref. [24], in many cases of interest, with an appropriate choice of integral basis, the coefficient matrix $A_{m}$ in eq. (2) becomes proportional to $\epsilon$. As a result, the basis integrals are manifestly expressed as iterated integrals. Refs. [25, 26] provide algorithms for finding a transformation to a canonical basis, which applies provided that a rational transformation exists 1

In many realistic multi-scale problems, such as $2 \rightarrow n$ scattering amplitudes with $n \geq 2$, the step of generating IBP reductions with existing algorithms is the most challenging part of the calculation. It is therefore of interest to explore other methods for generating these reductions.

In ref. [30] a subset of the present authors showed how IBP reductions that involve no squared propagators can be obtained efficiently on specific (algorithmically determined) sets of generalized-unitarity cuts. A similar approach was introduced by Harald Ita in ref. [31] where IBP relations are also studied in connection with cuts, and the underlying geometric interpretation is clarified.

In this paper we introduce the Singular [32]/Mathematica package AzURITE (A ZURich-bred method for finding master InTEgrals) which determines a basis for the space of integrals spanned by a given $L$-loop diagram and all of its subdiagrams (obtained by shrinking propagators). AzURITE can also be used to analytically generate IBP identities evaluated on maximal cuts.

In practice, the current version of this package can determine a basis of integrals for a two-loop diagram and all of its subdiagrams (no matter whether massless or massive, planar or non-planar) in seconds. It can also

\footnotetext{
${ }^{1}$ For some cases, the leading singularities are elliptic. Using complete elliptic integrals, these differential equations can be solved as iterated integrals with elliptic kernels [27, 28, 29 .
} 
determine master integrals for a three-loop diagram and all of its subdiagrams in minutes.

Related work has appeared in ref. [33] where the number of basis integrals is determined from the critical points of the polynomials that enter the parametric representation, or equivalently the Baikov representation, of the integral. This method has moreover been implemented in the Mathematica package Mint.

\section{Algorithm}

The algorithm of AzURITE may be summarized as follows: given an input diagram, the code traces over all subdiagrams and

1. automatically determines the automorphism group of the involved Feynman diagrams by graph theory algorithms,

2. detects and discards scaleless integrals (for example, diagrams with massless tadpoles),

3. determines the linear relations between integrals evaluated on maximal cuts for each subdiagram, using the methods of ref. [30] of constructing the IBP identities on $D$-dimensional generalized-unitarity cuts and solving syzygy equations. The on-shell version of the IBP identities, which have been constructed so as to contain no integrals with higherpower propagators, are generated numerically via finite field computations in Singular.

After these steps, AzURITE chooses a basis of integrals according to the following conventions: it removes all edge-reducible integrals from the candidate list of master integrals. (An edge-reducible integral is an integral which can be expressed as a linear combination of integrals from its subdiagrams.) For the remaining integrals, AzURITE considers IBP relations between integrals with different numerators, and finds a linear basis of integrals which contains the lowest possible numerator degrees. Only IBP identities evaluated on cuts are needed for determining the basis of integrals.

In the following we will explain the above steps in greater detail. To this end, we first introduce notation and some parametrizations of the integrals. We consider a general $L$-loop Feynman diagram with $n$ external lines, $k$ propagators, and all of its subdiagrams. The associated Feynman integrals 
are,

$$
I\left[a_{1}, \ldots, a_{k} ; N\right] \equiv \int \prod_{j=1}^{L}\left(\frac{\mathrm{d}^{D} l_{j}}{i \pi^{D / 2}}\right) \frac{N\left(l_{1}, \ldots, l_{L}\right)}{D_{1}^{a_{1}} \cdots D_{k}^{a_{k}}} .
$$

Let $k_{1}, \ldots, k_{n}$ be the external momenta, and $l_{1}, \ldots, l_{L}$ be the loop momenta. Following ref. [16], we restrict attention to IBP identities that do not involve integrals with higher-power propagators. Moreover, we will ultimately choose bases which do not contain such integrals, but rather contain integrals with numerator insertions. Therefore we require for the indices that $a_{i} \in\{0,1\}, i=$ $1, \ldots, k$. To simplify the notation, we denote

$$
\left\langle s_{1} \ldots s_{m}\right\rangle[N] \equiv \int \prod_{j=1}^{L}\left(\frac{\mathrm{d}^{D} l_{j}}{i \pi^{D / 2}}\right) \frac{N\left(l_{1}, \ldots, l_{L}\right)}{D_{s_{1}} \cdots D_{s_{m}}},
$$

where $1 \leq s_{1}<s_{2}<\cdots<s_{m} \leq k$ are the indices for existing propagators. We moreover use $\left\langle s_{1} \ldots s_{m}\right\rangle$ to denote the topology of the corresponding subdiagram.

The inverse propagators take the generic form,

$$
D_{i}=\left(\sum_{j=1}^{L} \alpha_{i j} l_{j}+\sum_{h=1}^{n} \beta_{i h} k_{h}\right)^{2}-m_{i}^{2} \equiv v_{i}^{2}-m_{i}^{2},
$$

where the $\alpha_{i j}$ and $\beta_{i h}$ are $\pm 1 . v_{i}$ denotes the momentum of the corresponding line.

We use dimensional regularization and work in the four-dimensional helicity scheme, taking the external momenta to be strictly four-dimensional. Accordingly, we decompose the loop momenta into four- and $(D-4)$-dimensional parts, $l_{i}=\bar{l}_{i}+l_{i}^{\perp}$. As explained in section 2 of ref. [30], for $n \leq 4$, the external momenta span a vector space of dimension less than four, and the components of the loop momenta along the orthogonal directions can be integrated out directly. After having done so, there are

$$
n_{\mathrm{SP}}=\phi(n) L+\frac{L(L+1)}{2},
$$

independent scalar products involving the loop momenta, where

$$
\phi(n) \equiv\left\{\begin{array}{cc}
4 & n \geq 5 \\
n-1 & n \leq 4
\end{array}\right.
$$


An application of the Ossola-Papadopoulos-Pittau (OPP) reduction method [34, 35, 36, 37, 38, 39], or integrand reduction via polynomial division wrt. Gröbner bases [40, 41], shows that if the number of distinct propagators is greater than the number of independent scalar products; i.e., $k>n_{\mathrm{SP}}$, then the diagram is reducible at the integrand level. Hence we can assume without loss of generality that $k \leq n_{\mathrm{SP}}$.

An important tool used in AzURITE is the Baikov representation [42] of an integral,

$$
\langle 12 \ldots k\rangle[N] \propto \int \mathrm{d} z_{1} \cdots \mathrm{d} z_{n_{\mathrm{SP}}} F\left(z_{1}, \ldots, z_{n_{\mathrm{SP}}}\right)^{\frac{D-h}{2}} \frac{N\left(z_{1}, \ldots, z_{n_{\mathrm{SP}}}\right)}{z_{1} \cdots z_{m}},
$$

where the $z_{1}, \ldots, z_{m}$ denote the inverse propagators $D_{s_{1}}, \ldots, D_{s_{m}} \cdot z_{m+1}, \ldots, z_{n_{\mathrm{SP}}^{\prime}}$ denote irreducible scalar products (ISPs, i.e., terms appearing in the numerator which cannot be written as linear combinations of inverse propagators). The quantity $F \equiv \operatorname{det}_{i, j} \mu_{i j}, i, j=1, \ldots, L$ appearing in the measure factor is occasionally referred to as the Baikov polynomial, whereas the exponent is defined as $h \equiv L+\phi(n)$. Here

$$
\mu_{i j} \equiv-l_{i}^{\perp} \cdot l_{j}^{\perp}
$$

where $l_{i}^{\perp}$ is the $(-2 \epsilon)$-dimensional component of $l_{i}$.

This representation is particularly suitable for generating IBP identities on generalized-unitarity cuts, and was used in refs. [31, 30]. Azurite computes $F$ through an appropriate change of variables of the loop momenta. It first parametrizes the loop momenta via van Neerven-Vermaseren coordinates [43, then separates the $\mu_{i j}$ and finally obtains the Baikov representation. The overall prefactor and the region of integration in eq. (8) are irrelevant for deriving IBP identities, and hence we neglect these. (The expressions for the overall pre-factor of the Baikov representation can be found in ref. [44].)

\subsection{Associated graphs and their symmetries}

Given the propagators in eq. (5), it is useful to obtain the corresponding graph algorithmically -i.e., to determine the vertices - for the purpose

of finding the discrete symmetries. This can be achieved by a backtracking algorithm. Define the set of flows of momenta on external and internal lines,

$$
M=\left\{k_{1}, \ldots, k_{n}, v_{1}, \ldots, v_{k},-v_{1}, \ldots,-v_{k}\right\}
$$


Search through the subsets of $M$ until finding a subset $V_{1}$ containing at least three entries and satisfying momentum conservation, $\sum_{p \in V_{1}} p=0$. $V_{1}$ is the candidate for the first vertex. Now redefine $M:=M-V_{1}$ and search through the subsets of $M$ to find $V_{2}$ analogously. Iterate this process. If, at some step, no $V_{i}$ can be found, then backtrack and redefine $M:=M \cup V_{i-1}$ and proceed to find a new candidate $V_{i-1}^{\prime}$ for the previous vertex. When $n_{V}$ vertices have been found, and the resulting graph is connected and has $L$ loops, the algorithm terminates. Here,

$$
n_{V}=k-L+1
$$

denotes the number of vertices (cf. section II.3 of ref. [45]).

As an example let us consider $L=2$ and the following eight inverse propagators,

$$
\begin{array}{llll}
D_{1}=l_{1}^{2}, & D_{2}=\left(l_{1}-k_{1}\right)^{2}, & D_{3}=\left(l_{1}-K_{12}\right)^{2}, & D_{4}=\left(l_{1}-K_{123}\right)^{2}, \\
D_{5}=\left(l_{2}+K_{123}\right)^{2}, & D_{6}=\left(l_{2}-k_{5}\right)^{2}, & D_{7}=l_{2}^{2}, & D_{8}=\left(l_{1}+l_{2}\right)^{2} .
\end{array}
$$

where $K_{i_{1} \cdots i_{s}} \equiv k_{i_{1}}+\cdots+k_{i_{s}}$. The backtracking method finds the vertices,

$$
\begin{array}{ll}
V_{1}=\left\{k_{1},-l_{1}, l_{1}-k_{1}\right\}, & V_{2}=\left\{k_{2}, l_{1}-K_{12},-l_{1}+k_{1}\right\}, \\
V_{3}=\left\{k_{3}, l_{1}-K_{123},-l_{1}+K_{12}\right\}, & V_{4}=\left\{k_{4}, l_{2}+K_{123},-l_{2}+k_{5}\right\}, \\
V_{5}=\left\{k_{5},-l_{2}, l_{2}-k_{5}\right\}, & V_{6}=\left\{-\left(l_{1}+l_{2}\right), l_{1}, l_{2}\right\}, \\
V_{7}=\left\{-l_{1}+K_{123},-\left(l_{2}+K_{123}\right), l_{1}+l_{2}\right\} . &
\end{array}
$$

From this information it is straightforward to construct the adjacency matrix of the graph. The graph is found to be the pentagon-box diagram illustrated in fig. 1 a.

Once the graph for $\langle 12 \ldots k\rangle$ has been found, all of its subdiagrams can be obtained by pinching subsets of its propagators. Taking a graph theoretical viewpoint, we obtain the various subdiagrams by appropriately truncating the adjacency matrix of the original graph. ${ }^{2}$ For example, $\langle 12345678\rangle$ in fig. $1 \mathrm{a}$ corresponds to a pentagon-box diagram, and $\langle 145678\rangle$ in fig. $1 \mathrm{~b}$ corresponds to to a triangle-box subdiagram.

\footnotetext{
${ }^{2}$ For example, if the edge $e$ connecting two vertices $v_{1}$ and $v_{2}$ is pinched, then we merge the two columns (and also the two rows) in the adjacency matrix that correspond to $v_{1}$ and $v_{2}$.
} 


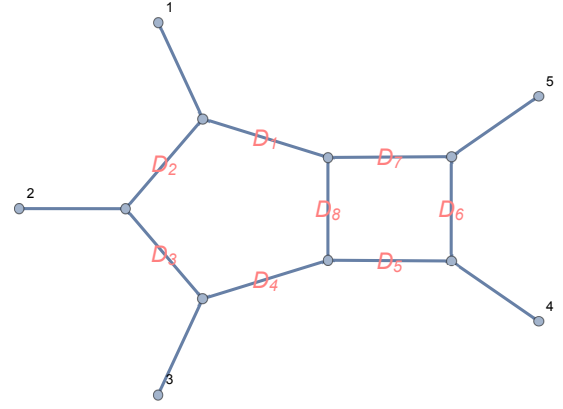

(a) The pentagon-box diagram $\langle 12345678\rangle$.

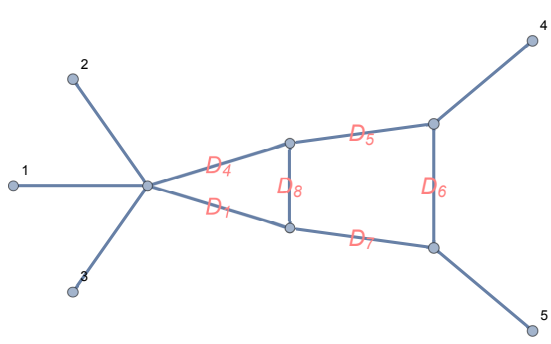

(b) The $\langle 145678\rangle$ subdiagram of the pentagon-box diagram.

Figure 1: Pentagon-box diagram and one of its subdiagrams.

Given a subdiagram $\left\langle s_{1} s_{2} \ldots s_{m}\right\rangle$, after having obtained its graph, we proceed to find its automorphism group $G^{\prime}$ via a graph theory based algorithm. $G^{\prime}$ acts on the propagators. Let $G$ denote the subgroup of $G^{\prime}$ which preserves all external Lorentz invariants. $G$ is the physical symmetry group of this diagram. $G$ actually classifies all subdiagrams of $\left\langle s_{1} s_{2} \ldots s_{m}\right\rangle$ into equivalence classes. (If two subdiagrams $g_{1}$ and $g_{2}$ are equivalent, then any integral with the topology $g_{1}$ must equal an integral with the topology $g_{2}$ with the appropriate numerator insertion.)

Furthermore, $G$ acts on momenta as affine transformations (linear and shift transformations). We explicitly find these transformations by linear algebra. This enables us to determine the action of $G$ on irreducible scalar products appearing in the numerator.

For instance, diagram $\langle 145678\rangle$ associated with the inverse propagators in eq. (B.3) has the symmetry group $G=Z_{2}$, whose non-trivial element is (cf. fig. 1b),

$$
D_{1} \mapsto D_{4}, \quad D_{4} \mapsto D_{1}, \quad D_{5} \mapsto D_{7}, \quad D_{7} \mapsto D_{5}, \quad D_{8} \mapsto D_{8}
$$

(Note that since both $k_{4}$ and $k_{5}$ are massless this symmetry preserves external Lorentz invariants, and hence is physical.) This implies symmetry relations for all of its subdiagrams. For example, by this symmetry, the integral $\langle 158\rangle$ is equal to $\langle 478\rangle$. Thus, we only need to consider $\langle 158\rangle$ during the search for master integrals, and can neglect $\langle 478\rangle$.

In this example, the non-trivial element of $G$ given in eq. (14) corresponds 
to the affine transformation,

$$
k_{4} \mapsto k_{5}, \quad k_{5} \mapsto k_{4}, \quad l_{1} \mapsto-k_{4}-k_{5}-l_{1}, \quad l_{2} \mapsto-k_{4}-k_{5}-l_{2}
$$

From this, the action of $G$ on numerator polynomials can readily be found.

This backtracking graph-construction algorithm is implemented in AzURITE, powered by MATHEMATICA. The graph automorphism groups, connectedness condition, and other graph information are computed via Mathematica's embedded graph commands $3^{3}$ The affine transformations such as those in eq. (15) are obtained by setting up an ansatz of the action on the momenta of the internal lines,

$$
v_{i} \mapsto c_{i} v_{g(i)}, \quad \forall i \in\left\{s_{1}, \ldots, s_{m}\right\}
$$

for $g \in G$. To ensure that this is a permutation of the propagators, all of the $c_{i}$ must be \pm 1 . Using standard linear algebra techniques, the values of the $c_{i}$ are readily solved for, and the affine transformation is determined.

\subsection{Adaptive parameterization and further graph simplifications}

To optimize the search for master integrals we apply the following simplifications during the study of the input diagram and all of its subdiagrams. (Similar simplifications for subdiagrams are used in the adaptive integrand decomposition approach of ref. [46].)

1. If a diagram has a loop which corresponds to a scaleless integral, then the diagram vanishes in dimensional regularization. For example, with the inverse propagators in eq. (B.3), the diagram $\langle 12346\rangle$ (illustrated in fig. 2a) contains a massless tadpole and hence vanishes. Azurite finds such loops by examining the fundamental cycles $4^{4}$ of the graph. Moreover, the diagram $\langle 1234\rangle$ corresponds to an integral without $l_{2}$ appearing in the denominator, so that the $l_{2}$ integral is scaleless and hence vanishes. Both of these diagrams are therefore discarded.

2. If for a diagram, two or more external lines attach to one vertex, we may combine these external lines into one external line with the sum of the individual momenta flowing on it. We let $n^{\prime}$ denote the number

\footnotetext{
${ }^{3}$ Mathematica 10.0 .0 or later versions are required for the graph theory computations in Azurite.

${ }^{4}$ See section II.3 of ref. 45] for the definition of fundamental cycles.
} 


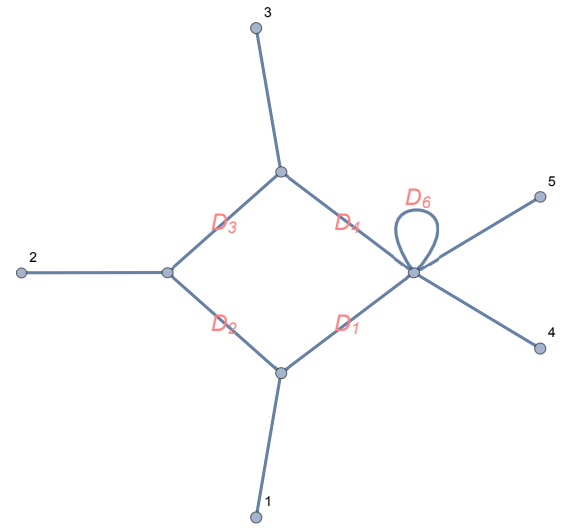

(a) The subdiagram $\langle 12346\rangle$, which contains a massless tad- (b) The subdiagram $\langle 1234567\rangle$, pole.

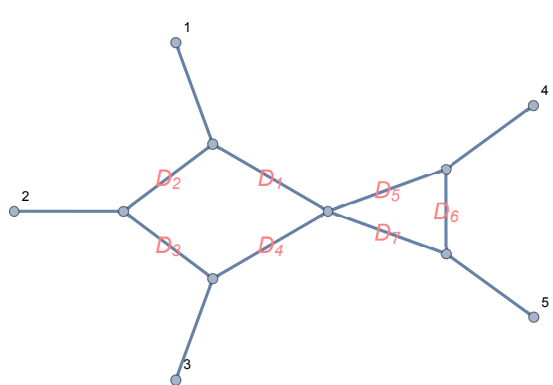

which is factorable.

Figure 2: Some diagrams which can be simplified in the adaptive parametrization.

of new external lines after this procedure, where clearly $n^{\prime}<n$. As an example, for the inverse propagators in eq. (B.3), the diagram $\langle 145678\rangle$ (illustrated in fig. 2b can be treated as a three-point diagram with the new external momenta $K_{123}, k_{4}$ and $k_{5}$. It may occasionally be necessary to shift the loop momenta to ensure that only the new external momenta appear in the propagators. This is achieved in AzURITE by linear algebra methods.

We also define $n_{\mathrm{SP}}^{\prime}=\phi\left(n^{\prime}\right) L+L(L+1) / 2$ as the number of new independent scalar products. For example, the diagram $\langle 145678\rangle$ (illustrated in fig. $2 \mathrm{~b}$ has $n_{\mathrm{SP}}^{\prime}=2 \times 2+3=7$. This process decreases the number of scalar products and thereby significantly speeds up the IBP computations.

3. If a diagram consists of $n_{\Gamma}\left(n_{\Gamma}>1\right)$ loops that do not share common edges, we call the diagram factorable and treat the corresponding integral as a product of $n_{\Gamma}$ integrals. For example, with the inverse propagators given in eq. (B.3), the diagram $\langle 1234567\rangle$ is treated as the product of two one-loop diagrams. This is achieved in AzURITE by examining the fundamental cycles of the graph.

For any subdiagram $\left\langle s_{1} \ldots s_{m}\right\rangle$ encountered we apply these simplifications. After doing so, the diagram has $n^{\prime}$ external lines and $n_{\mathrm{SP}}^{\prime}$ independent 
scalar products, and we can assume without loss of generality that the diagram is non-factorizable and contains no scaleless integrals. By integrand reduction, we furthermore have $m \leq n_{\mathrm{SP}}^{\prime}$. We then proceed to cast the corresponding integrals in their Baikov representation (8) (with the number of independent scalar products computed from the adaptive parametrization of the integral so that $n_{\mathrm{SP}} \rightarrow n_{\mathrm{SP}}^{\prime}$ and $\left.h=L+\phi\left(n^{\prime}\right)\right)$.

\subsection{IBP identities on maximal cuts and master integrals}

Given the input diagram $\langle 12 \ldots k\rangle$, let us denote the set which consists of $\langle 12 \ldots k\rangle$ and all of its subdiagrams as $\mathcal{S}^{\prime \prime}$. Using symmetries we identify equivalent diagrams within $\mathcal{S}^{\prime \prime}$ and obtain the subset $\mathcal{S}^{\prime} \subset \mathcal{S}^{\prime \prime}$ such that no two elements of $\mathcal{S}^{\prime}$ are equivalent by a discrete symmetry. $\mathcal{S}^{\prime}$ consists of candidate topologies for master integrals.

Furthermore, we discard diagrams in $\mathcal{S}^{\prime}$ with scaleless loops, and simplify diagrams by rewriting them in their adaptive representation if applicable, as described in the previous subsection. The set of remaining diagrams is denoted by $\mathcal{S}$. Then we cast the integrals in $\mathcal{S}$ in their Baikov representation (cf. eq. (8)).

There are many different ways of choosing a basis of integrals. AzURITE chooses the basis as follows: it prefers integrals with monomials in the numerator to integrals with higher-power propagators. Moreover, it prefers a choice of basis whose integrals have as few propagators as possible. This is to make the computation more efficient, as this convention facilitates the use of IBP relations evaluated on their maximal cuts. (Otherwise, we need complete IBP relations, without cuts applied, to find an integral basis.) Accordingly, as the code traces over the subdiagrams of the input diagram, it removes edgereducible integrals. These are integrals which can be expressed as a linear combination of integrals that correspond to strict subdiagrams. Evaluated on its maximal cut $D_{s_{1}}=\cdots=D_{s_{m}}=0$, an edge-reducible integral reads,

$$
\left\langle s_{1} \ldots s_{m}\right\rangle[N]=0+(\text { strict subdiagrams }),
$$

where the strict subdiagrams vanish on this cut. $N$ is a monomial of irreducible scalar products. Similarly, for the remaining integrals, we consider IBP identities without squared propagators (cf. ref. [16]) to find linear relations between integrals with different numerators. Again, evaluated on its maximal cut, a general IBP identity reads,

$$
\sum_{i} c_{i}\left\langle s_{1} \ldots s_{m}\right\rangle\left[N_{i}\right]=(\cdots)
$$


where each $N_{i}$ is a monomial, and $(\cdots)$ denotes integrals that correspond to strict subdiagrams. We moreover use the symmetry group $G$ of $\left\langle s_{1} \ldots s_{m}\right\rangle$ to find linear relations, taking a form similar to that of eq. (18). To find the linear basis of integrals, we introduce a monomial order $\succ$ for all monomials in the irreducible scalar products. After obtaining enough IBP and symmetry relations, we linearly reduce integrals according to $\succ$ via Gaussian elimination. In practice, a good choice of $\succ$ is either degree reverse lexicographic or degree lexicographic order, as this ensures that the chosen basis contains monomials with as low degree as possible. (In contrast, lexicographic monomial order may lead to high-degree numerators.)

AzURITE traces through all diagrams in $\mathcal{S}$ and obtains the complete list of master integrals. Because of the nature of sub-diagrams, this computation can be finished in a parallelized way. Only IBP identities evaluated on their maximal cuts and symmetry relations are needed to find a basis. Hence we focus our attention to obtaining eqs. (17) and (18), i.e., IBP identities evaluated on their maximal cut. The representation in eq. (8) can easily accommodate the maximal cut of any subdiagram by taking the residue at $z_{1}=\cdots=z_{m}=0$, (adaptive parametrization is used so that $z_{1}, \ldots, z_{m}$ denote propagators and $z_{m+1}, \ldots, z_{n_{\mathrm{SP}}^{\prime}}$ denote ISPs)

$$
\begin{gathered}
\left.\left\langle s_{1} \ldots s_{m}\right\rangle[N]\right|_{\text {maximal cut }} \propto \int \mathrm{d} z_{m+1} \cdots \mathrm{d} z_{n_{\mathrm{SP}}^{\prime}} F\left(0, \ldots, 0, z_{m+1}, \ldots, z_{n_{\mathrm{SP}}^{\prime}}\right)^{\frac{D-h}{2}} \\
\times N\left(0, \ldots, 0, z_{m+1}, \ldots, z_{n_{\mathrm{SP}}^{\prime}}\right) .
\end{gathered}
$$

Again we neglect the overall prefactor and the region of integration. Define $f\left(z_{m+1}, \ldots, z_{n_{\mathrm{SP}}^{\prime}}\right) \equiv F\left(0, \ldots, 0, z_{m+1}, \ldots, z_{n_{\mathrm{SP}}^{\prime}}\right)$. Cf. refs. [31, 30], IBP identities evaluated on their maximal cut take the following form ${ }^{5}$

$$
\begin{aligned}
0 & =\int \mathrm{d} z_{m+1} \cdots \mathrm{d} z_{n_{\mathrm{SP}}^{\prime}} \sum_{i=m+1}^{n_{\mathrm{SP}}^{\prime}} \frac{\partial}{\partial z_{i}}\left(a_{i}\left(z_{m+1}, \ldots, z_{n_{\mathrm{SP}}^{\prime}}\right) f\left(z_{m+1}, \ldots, z_{n_{\mathrm{SP}}^{\prime}}\right)^{\frac{D-h}{2}}\right) \\
& =\int \mathrm{d} z_{m+1} \cdots \mathrm{d} z_{n_{\mathrm{SP}}^{\prime}}\left(f^{\frac{D-h}{2}} \sum_{i=m+1}^{n_{\mathrm{SP}}^{\prime}} \frac{\partial a_{i}}{\partial z_{i}}+\frac{D-h}{2} f^{\frac{D-h-2}{2}} \sum_{i=m+1}^{n_{\mathrm{SP}}^{\prime}} a_{i} \frac{\partial f}{\partial z_{i}}\right) .
\end{aligned}
$$

\footnotetext{
${ }^{5}$ Note that the third term of the general form given in eq. (11) of ref. [30] is absent on the maximal cut where the number of cuts is equal to the number of propagators, $c=k$.
} 
Here the $a_{i}$ (a priori) are arbitrary polynomials in the ISPs $z_{m+1}, \ldots, z_{n_{\mathrm{SP}}^{\prime}}$. The second term in eq. (21) corresponds to integrals in $D-2$ dimensions. To compensate this shift we require,

$$
\sum_{i=m+1}^{n_{\mathrm{SP}}^{\prime}} a_{i} \frac{\partial f}{\partial z_{i}}+a f=0
$$

where $a$ is a polynomial in the ISPs. Equations of this kind are known in algebraic geometry as syzygy equations. Syzygy equations were also used for deriving IBP identities for integrals in Feynman parametrization [47]. The current version of AzURITE uses the command syz in Singular to find all generators of the solution set of eq. (22).

Then the IBP identity evaluated on its maximal cut reads,

$$
0=\int \mathrm{d} z_{m+1} \cdots \mathrm{d} z_{n_{\mathrm{SP}}^{\prime}} f^{\frac{D-h}{2}}\left(\sum_{i=m+1}^{n_{\mathrm{SP}}^{\prime}} \frac{\partial a_{i}}{\partial z_{i}}-\frac{D-h}{2} a\right)
$$

or equivalently,

$$
\left\langle s_{1} \ldots s_{m}\right\rangle\left[\sum_{i=m+1}^{n_{\mathrm{SP}}^{\prime}} \frac{\partial a_{i}}{\partial z_{i}}-\frac{D-h}{2} a\right]=(\cdots),
$$

where $(\cdots)$ denotes integrals that correspond to strict subdiagrams.

In practice, given the generators of the syzygy module,

$$
\mathbf{g}^{(j)}=\left(a_{m+1}^{(j)}, \ldots a_{n_{\mathrm{SP}}^{\prime}}^{(j)}, a^{(j)}\right)
$$

we need to consider the syzygy $\left(a_{m+1}, \ldots a_{n_{\mathrm{SP}}^{\prime}}, a\right)=P \mathbf{g}^{(j)}$ for the IBP formula (23). Here $P$ is an arbitrary polynomial in the ISPs, with the degree up to a fixed integer.

AzURITE generates IBP identities evaluated on their maximal cut by the use of eq. (23) allowing IBP identities up to a maximum degree. For the Gaussian elimination step, it lists the coefficients of monomials in each IBP identity in a monomial order of ISPs (degree reverse lexicographic by default). In this way a matrix of IBP coefficients is obtained. Then by Gaussian elimination of this matrix, independent IBP identities are identified. The master integrals correspond to the non-pivot columns of the reduced matrix. 
The current version of AzURITE uses slimgb in Singular, which applies fast sparse linear algebra algorithms to carry out Gaussian elimination. The integral basis search can be parallelized for the sub-diagrams in $\mathcal{S}$, via the command ParallelTable in Mathematica.

For the purpose of finding a basis of integrals, numerical values for the external kinematic invariants and spacetime dimension suffice. Using in addition finite field techniques, this has the benefit of speeding up the computation of syzygies and the Gauss-Jordan elimination step. In some cases analytic IBP identities evaluated on maximal cuts are useful, for instance for the study of multi-loop maximal unitarity in integer spacetime dimensions $[48,49,50,51,52,53]$. In this case analytic kinematics and spacetime dimension $D$ would be used by AzURITE for generating analytic IBP identities on the maximal cut.

\subsection{Geometric interpretation of syzygy equation}

In this subsection we digress from the mainstream of the text to discuss a geometric interpretation of the constraint (22). The geometric picture of syzygies evaluated on unitarity cuts was first discussed in ref. 31]. Here we reformulate the geometric interpretation in tangent algebra language.

The basic observation is that the polynomial-valued vector field

$$
\sum_{i=m+1}^{n_{\mathrm{SP}}^{\prime}} a_{i} \frac{\partial}{\partial z_{i}}
$$

is tangent to the hypersurface defined by $f\left(z_{m+1}, \ldots, z_{n_{\mathrm{SP}}^{\prime}}\right)=0$ [31]. The solution set of eq. (22) is the module of syzygies,

$$
\operatorname{syz}\left(\frac{\partial f}{\partial z_{m+1}}, \ldots, \frac{\partial f}{\partial z_{n_{\mathrm{SP}}^{\prime}}}, f\right) .
$$

The $\left(a_{m+1}, \ldots, a_{n_{\mathrm{SP}}^{\prime}}\right)$ from this syzygy module form the module of the tangent algebra $\mathbf{T}_{f}$ [54], i.e., the set of all polynomial-valued tangent vector fields for the hypersurface $f=0$. $\mathbf{T}_{f}$ is a Lie algebra and infinite-dimensional in general.

The structure of $\mathbf{T}_{f}$ depends on the geometric properties of the hypersurface $f=0$. For example, when the hypersurface is non-singular, i.e., the singular ideal $I_{s}$ satisfies

$$
I_{s} \equiv\left\langle\frac{\partial f}{\partial z_{m+1}}, \ldots, \frac{\partial f}{\partial z_{n_{\mathrm{SP}}^{\prime}}}, f\right\rangle=\langle 1\rangle,
$$


then the solution of eq. 22 is generated by principal syzygies (trivial syzygy relations) [55. This can be proven by multiplying any syzygy relation by "1", and replacing " 1 " by the generators of the singular ideal in eq. (28). In this case, no computation is needed for obtaining the generators of $\mathbf{T}_{f}$.

If the hypersurface $f=0$ is singular, then locally around a singular point, $\mathbf{T}_{f}$ is generated by principal syzygies and weighted Euler vectors [54]. Moreover, cf. Schreyer's theorem [55], the generators of the solutions of eq. (22) can be found algebraically via S-polynomial computations.

\section{Examples and performance}

In this section we present some non-trivial results obtained from AzURITE along with some benchmarks of its performance. An introduction to the functions and their usage can be found in Appendix A. In all of the following cases, the full numerical approach is used $!^{6}$ This setup is the most computationally favourable for SingulaR.

As an example, let us consider the triple-box diagram with $k=10$ propagators illustrated in the top row of fig. 3. First we present the initialization of AzURITE for this diagram, shown in the sample code 1 .

The list of loop momenta is declared in LoopMomenta. A list of linearly independent momenta is declared in ExternalMomenta. The list Propagators consists of the propagators of the diagram, augmented by a list of the independent ISPs. They are found by enumerating all the possible scalar products involving the loop momenta, and by finding a maximum-rank subset. In the case at hand there are, cf. eq. (6), $n_{\mathrm{SP}}-k=15-10=5$ independent ISPs. These are the last five elements of Propagators below. In Kinematics, Lorentz invariants formed of external momenta are expressed in terms of the Mandelstam invariants. In Numerics, numerical values are given for the kinematical invariants. These must be chosen randomly, so as to avoid poles in the intermediate reduction steps.

Having declared the diagram, we can now proceed to compute the master integrals of the vector space spanned by this diagram and its subdiagrams. This is done with the FindAllmIs function, where the first input entry of FindAllMIs (sample code 2) is a list of labels of the propagators of the di-

\footnotetext{
${ }^{6}$ The computations were carried out on a i7-6700, 32GB DDR4 RAM machine using SingULAR v4.0.3, with parallel computations.
} 


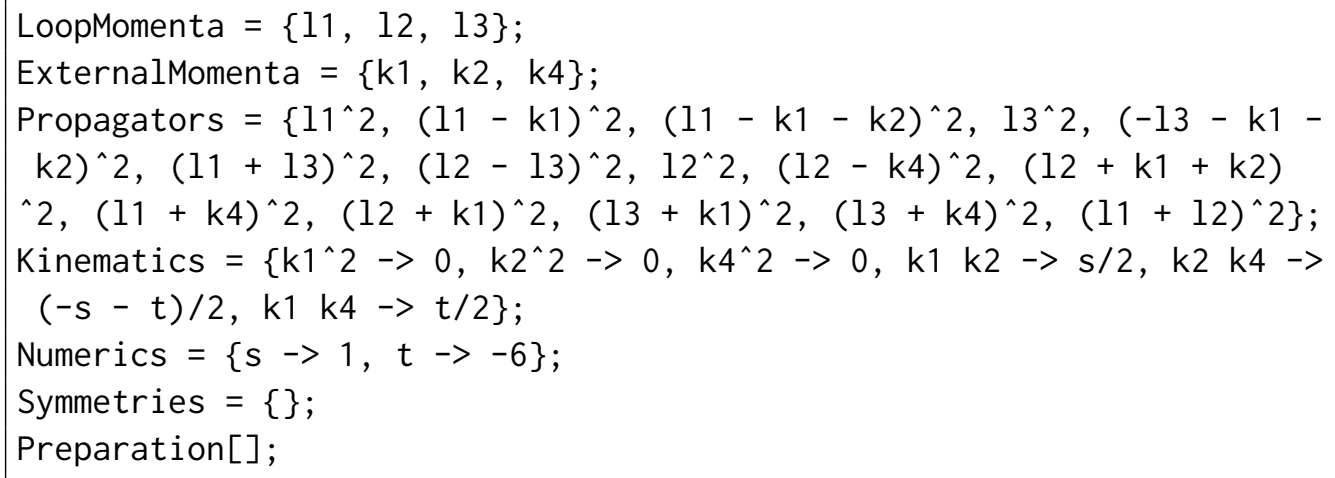

Azurite sample code 1: Initialization for a massless triple-box diagram.

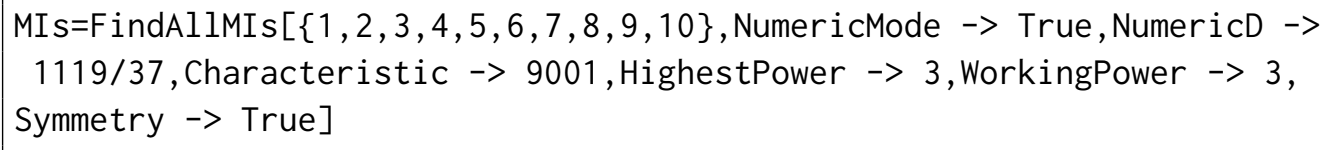

Azurite sample code 2: Example of use for FindAllMIs.

agram. FindAllMIs can be used with the parallel computation. We refer to section Appendix A.2.4 for further details on the syntax.

The computation is performed by making use of adaptive parametrizations (cf. section 2.2) of all the subdiagrams encountered in the IBP relations that are generated, and taking into account their discrete symmetries (cf. section 2.1). With the options chosen above, the computation is moreover performed in a finite field of characteristic 9001 and with the numerical value of $\frac{1119}{37}$ for the space-time dimension, chosen such that there are no dimension dependent poles in the reduction coefficients.

The total time elapsed for the complete reduction is, on our desktop computer with parallel computation, 68 seconds. The irreducible topologies that are chosen as a basis are shown in fig. 3. Their respective graphs were drawn using the function FeynmanGraph. 


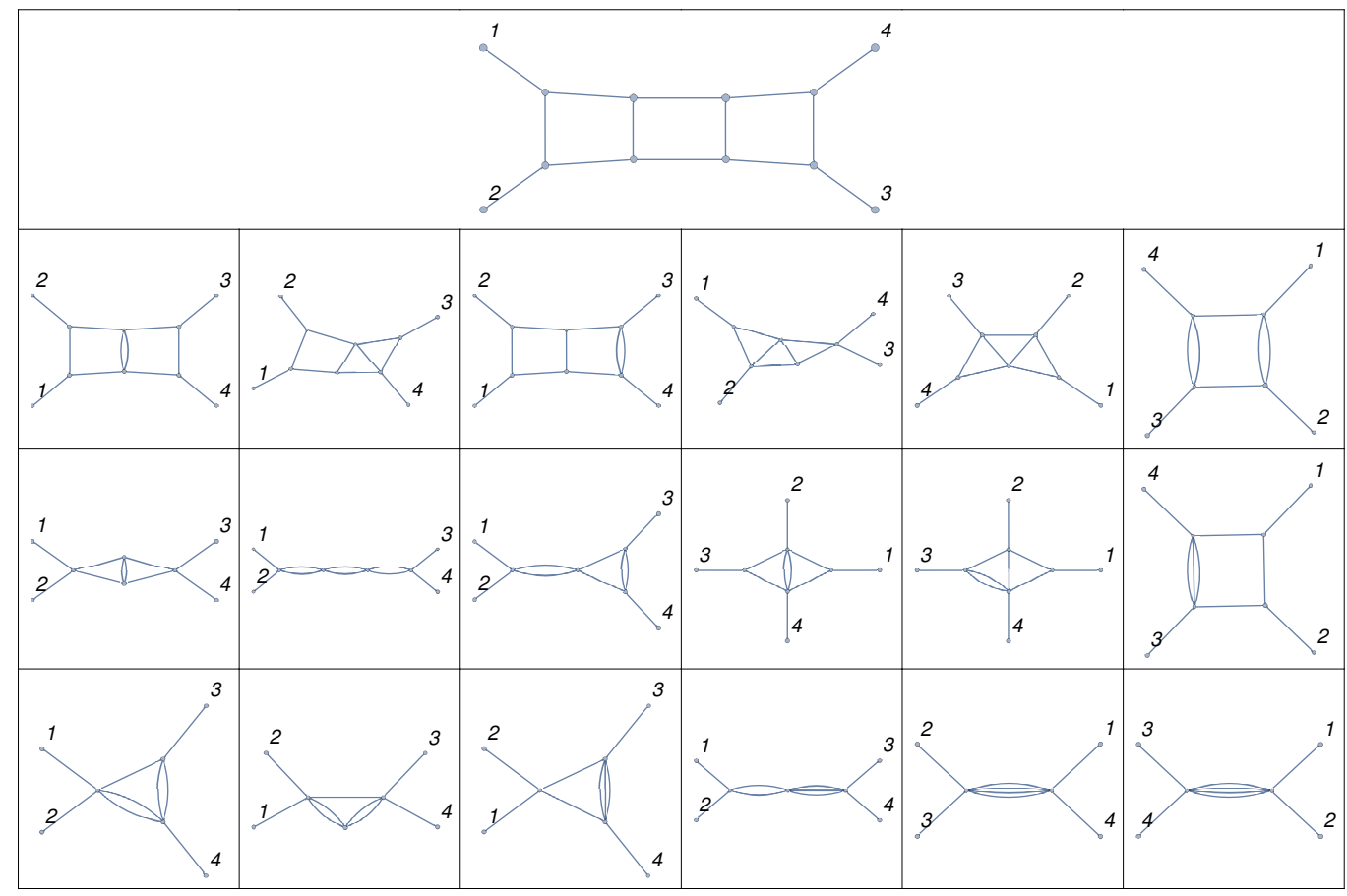

Figure 3: Irreducible topologies for the massless triple-box diagram.

Using the notation of eq. (8) we can rewrite the 26 basis elements as

$$
\begin{aligned}
& \langle 12345678910\rangle\left[z_{11}, z_{13}, 1\right] \quad\langle 123678910\rangle\left[z_{4}, 1\right] \quad\langle 123467910\rangle\left[z_{5}, 1\right] \\
& \langle 12345679\rangle\left[z_{8}, 1\right] \quad\langle 12567810\rangle[1] \quad\langle 1256789\rangle\left[z_{3}, 1\right] \quad\langle 245679\rangle\left[z_{1}, 1\right] \\
& \langle 1367810\rangle[1] \quad\langle 1345810\rangle[1] \quad\langle 134579\rangle[1] \quad\langle 1267910\rangle[1] \quad\langle 125679\rangle[1] \\
& \langle 123679\rangle[1] \quad\langle 15679\rangle[1] \quad\langle 15678\rangle[1] \quad\langle 13679\rangle[1] \quad\langle 134710\rangle[1] \\
& \langle 2679\rangle[1] \quad\langle 167 \text { 10 [1], }
\end{aligned}
$$

where the values inside the square brackets represent the irreducible numerators for the given topology. The numerators are expressed in the Baikov representation using the variables $z_{i}$. Here $i$ is an integer corresponding to the $i$ th element of the list Propagators. The possible values of $i$ are determined by the uncut propagators, for example for the $\langle 123678910\rangle$ subdiagram the numerators can be chosen as:

$$
\begin{array}{lll}
z_{4}=\left(l_{3}\right)^{2}, & z_{5}=\left(l_{3}+k_{1}+k_{2}\right)^{2}, & z_{11}=\left(l_{1}+k_{4}\right)^{2}, \\
z_{12}=\left(l_{2}+k_{1}\right)^{2}, & z_{13}=\left(l_{3}+k_{1}\right)^{2}, & z_{14}=\left(l_{3}+k_{4}\right)^{2} \\
z_{15}=\left(l_{1}+l_{2}\right)^{2} & &
\end{array}
$$


Other than the 5 initial ISPs the two uncut denominators $\left\{z_{4}, z_{5}\right\}$ can appear as numerators.

The reduction is very efficient. This is evidenced in fig. 4 which displays results for a variety of diagrams at various loop orders and configurations of internal and external masses? Here, $\boldsymbol{\Lambda}, \mathbf{\square}$ and $\bullet$ represent different masses.

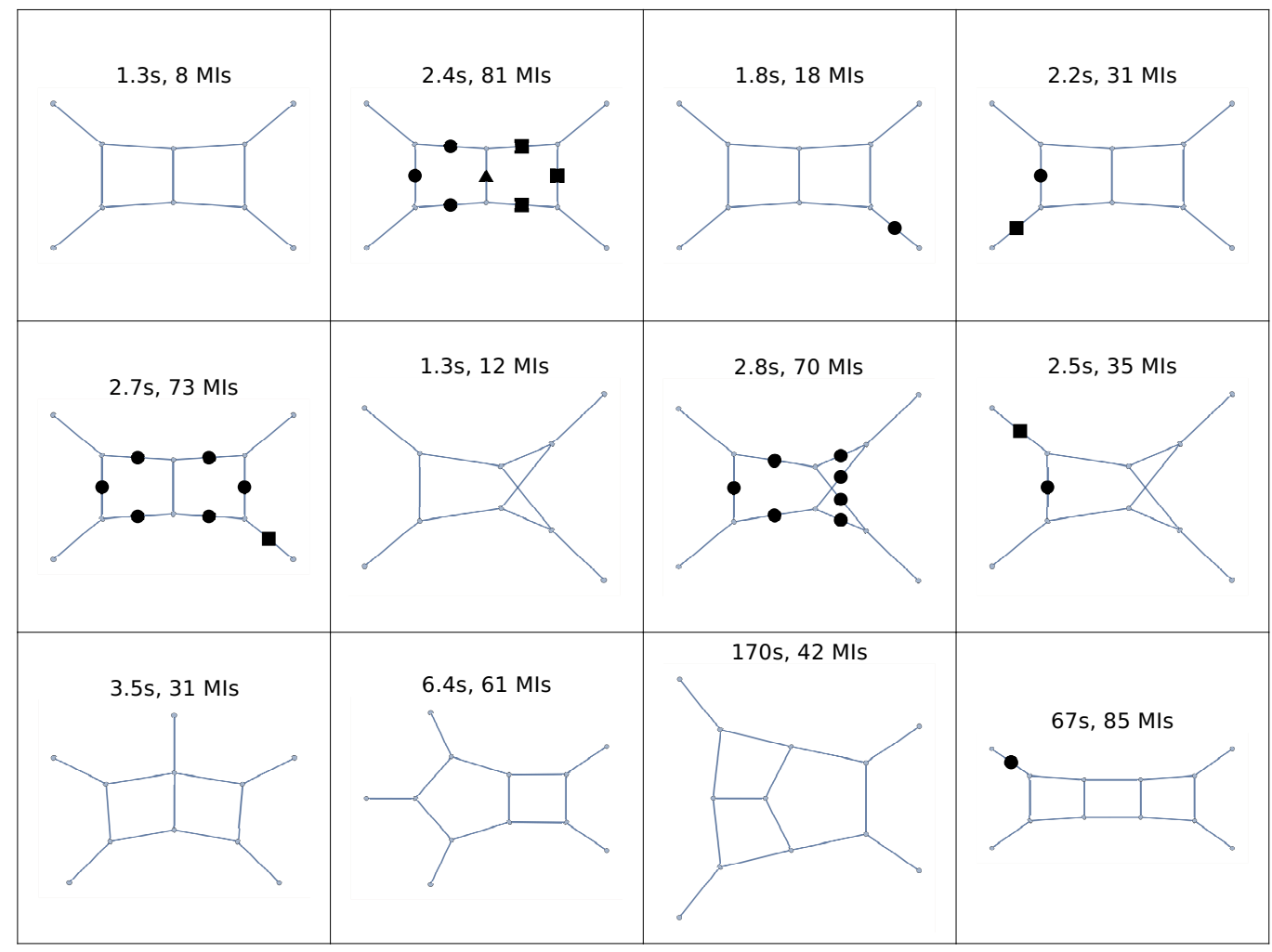

Figure 4: Computation time and number of master integrals for different topologies and mass configurations.

\subsection{IBP identities evaluated on their maximal cut}

AzURITE can also obtain IBP identities on maximal cuts, both analytically or numerically, using the function IntegralRed. For example, for the triple-box diagram (Azurite sample code 1), the analytic IBP identities on

\footnotetext{
${ }^{7}$ Part of these results were already known, and our results agree with the literature, see for example refs. $26,28,56$.
} 
IntegralRed $[\{1,2,3,4,5,6,7,8,9,10\}]$;

Azurite sample code 3: Sample code for IntegralRed

the maximal cut $D_{1}=D_{2}=\ldots=D_{10}=0$ can be obtained. The output is a list whose first element contains the master integrals and the second one contains the IBP identities evaluated on the cut, in the form of replacement rules.

Using a similar notation as in eq. (29) we can represent the master integrals for this cut as $\left\{\mathrm{I}\left[z_{11}\right], \mathrm{I}\left[z_{13}\right], \mathrm{I}[1]\right\}$. Here the prescription $\mathrm{I}[\mathrm{N}]$ indicates, using again the Baikov representation,

$$
\mathrm{I}[\mathrm{N}]=\int \prod_{i=11}^{15} \mathrm{~d} z_{i} F^{\frac{d-7}{2}} \mathrm{~N}
$$

The values of $i$ run over the ISPs of this diagram, which can be read off from eq. (30). For instance, the reduction of $\mathrm{I}\left[z_{11}^{2}\right]$ is then written as

$$
\begin{aligned}
\mathrm{I}\left[z_{11}^{2}\right]=\frac{1}{2(-3+d)^{2}} & \left(-2\left(\left(8-6 d+d^{2}\right) s-(-3+d) t\right) \mathrm{I}\left[z_{11}\right]\right. \\
& \left.+2(-4+d)^{2} t \mathrm{I}\left[z_{13}\right]+(-4+d)(-2+d) \text { st } \mathrm{I}[1]\right)+\ldots
\end{aligned}
$$

where ... denotes integrals with fewer-than-ten propagators. It takes about 2.4 seconds to reduce all numerators up to rank 4 to the master integrals, and about 18.0 seconds to reduce all numerators up to rank 6 to the master integrals, on the maximal cut, with the same computer mentioned in the previous subsection.

\section{Summary and Outlook}

In this paper, we have introduced our new algorithm for finding bases of loop integrals and its implementation in the package AzURITE. It constructs the needed integration-by-parts identities on a specific set of (algorithmically determined) cuts, and constructs identities where integrals with higher-power propagators are absent by solving syzygy equations. By making use of further simplifications, involving adaptive parametrizations of the involved diagrams, 
using graph theory tools to find discrete symmetries, finite-field computations and parallel computations, the package finds master integrals for two- and three-loop diagrams very efficiently. Therefore we expect that AzURITE will be a very useful tool for studies of multi-loop scattering amplitudes, for example in IBP reductions and differential equations. This package can also be used to find the IBP relations evaluated on their maximal cuts analytically.

There are several directions for developing new versions of AzURITE. One direction is to write a new syzygy generating code, based on new developments in computational algebraic geometry such as Faugère's F5 algorithm [57. The goal is to get the code to produce a simpler form of syzygy generators, which would allow speeding up the search for master integrals. It will also be very helpful to fully incorporate the tangent Lie algebra/variety duality [54] for deriving syzygies. Furthermore, we are working on a public package to produce complete IBP reductions efficiently, based on the present algorithm to find a basis of integrals, and on the construction of IBP reductions on cuts via syzygy computations [31, 30].

\section{Acknowledgements}

We thank S. Badger, Z. Bern, J. Bosma, L. Dixon, C. Duhr, H. Frellesvig, J. Henn, H. Ita, H. Johansson, D. Kosower, A. von Manteuffel, F. Moriello, E. Panzer, C. Papadopoulos, R. Schabinger and M. Zeng for useful discussions. Especially, we thank S. Badger and H. Ita for testing our package and for careful reading of our manuscripts during the draft stage. The research leading to these results has received funding from the European Union Seventh Framework Programme (FP7/2007-2013) under grant agreement no. 627521, and Swiss National Science Foundation (Ambizione grant PZ00P2 161341). The work of AG is supported by the Knut and Alice Wallenberg Foundation under grant \#2015-0083. The work of AG and YZ is also partially supported by the Swiss National Science Foundation through the NCCR SwissMap. The work of KJL is supported by ERC-2014-CoG, Grant number 648630 IQFT.

\section{Appendix A. Usage of Azurite}

\section{Appendix A.1. Installation}

To install AzURITE, it is necessary to install the computer algebra systems Mathematica (10.0.0 or more recent versions) and Singular [32] 
first. Singular can be downloaded from http://www.singular.uni-kl.de. AZURITE version a.b.c can be downloaded from,

https://bitbucket.org/yzhphy/azurite/raw/master/release. Azurite_a.b.c.tar.gz

Here $a, b$ and $c$ must be replaced by the corresponding version numbers, for example,

https://bitbucket.org/yzhphy/azurite/raw/master/release!

Azurite_1.1.0.tar.gz.

After extracting the tar file Azurite_a.b.c.tar.gz, there will be a directory Azurite_a.b.c which consists of the sub-directories code, examples and manual. The main package file Azurite.wl is located in code. examples contains examples while manual contains a manual of AzURITE in MATHEMATICA notebook format.

A directory for temporary files must be created by the user.

Appendix A.2. Commands and Options

Appendix A.2.1. Path setup

The paths of AzURITE for temporary files and Singular binary file are set up as follows, in MATHEMATicA code. For example,

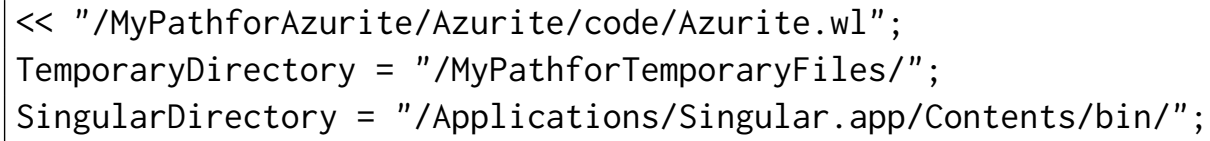

Here TemporaryDirectory denotes the directory of temporary files, while the variable SingularDirectory denotes the directory of the Singular binary file which depends on the operating system.

\section{Appendix A.2.2. Kinematics and loop structure information}

The loop structure and kinematics information should be added after the path set-up section. The names of loop momenta and external momenta are declared in LoopMomenta and ExternalMomenta, respectively. Inverse propagators, kinematics and numerical values of external invariants are listed in Propagators, Kinematics and Numerics respectively. The command Preparation[] finds the Baikov representation.

For example, the input for the pentagon-box in eq. (B.3) is, 


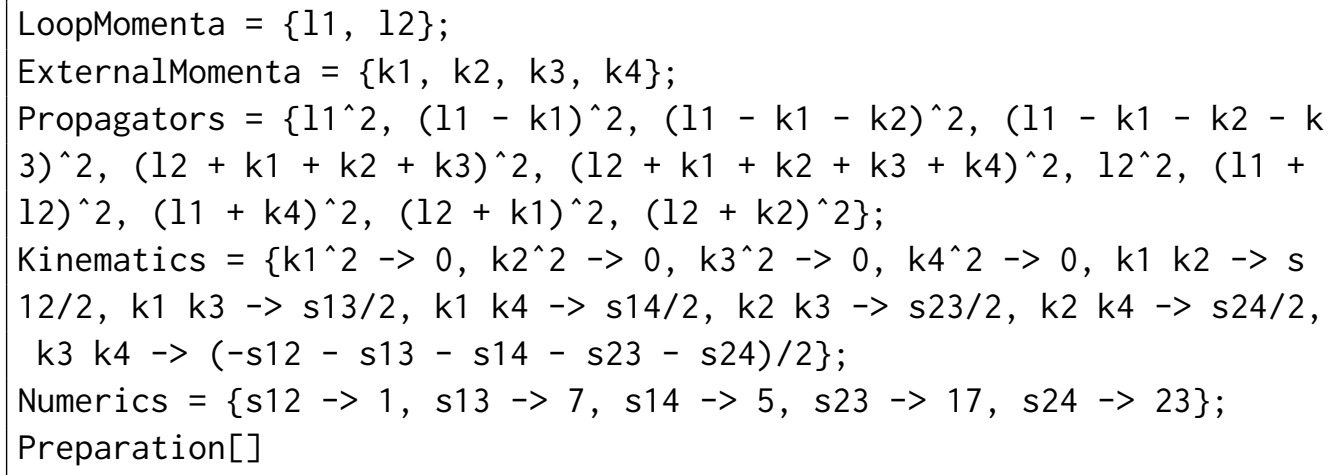

We have the following requirements,

- Only linearly independent external momenta can appear in ExternalMomenta and Kinematics. For example, we do not have $k_{5}$ in the input.

- The numerical input Numerics is necessary and the numerical values for kinematic variables should be generic. For example, the following input should be avoided, as the external mass $m_{1}$ is set to a non-generic value,

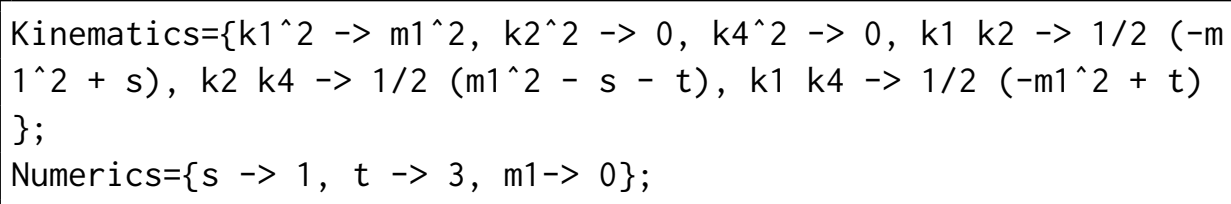

From the first line, Azurite will take $k_{1}$ to be massive for deriving the Baikov representation. However, $\mathrm{m} 1->0$ in second line may make the obtained Baikov representation singular. The correct input for a massless $k_{1}$ is,

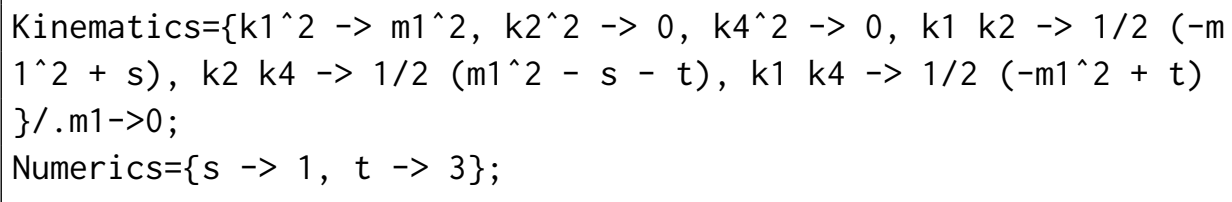

- Irreducible scalar products should be added to Propagators. The goal is to ensure that the elements in Propagators independently span the 
space of scalar products formed out of $l_{i} \cdot l_{j}$ and $l_{i} \cdot k_{j}$. (The $l_{i}$ are the loop momenta while the $k_{j}$ are the independent external momenta.)

\section{Appendix A.2.3. Associated graphs and their discrete symmetries}

After the preparation, AzURITE can find graphs and symmetries via the graph functions of MATHEMATICA. For example, with the inverse propagators given in eq. (B.3), the graphs of $\langle 12345678\rangle$ and $\langle 145678\rangle$ are obtained by calling,

FeynmanGraph[ $[1,2,3,4,5,6,7,8\}]$

FeynmanGraph $[\{1,4,5,6,7,8\}]$

FeynmanGraph has several options, including,

1. DiagramExtendedOutput with the default value False. If its value is True, then propagator labels will appear on the corresponding internal lines 8

2. FetchCachedGraphInfo with the default value True. To speed up the drawing of graphs, it is advantageous to first store the input diagram in RAM. This is achieved by calling DiagramCache, for example,

DiagramCache $[\{1,2,3,4,5,6,7,8\}]$;

Then, provided FetchCachedGraphInfo has the value True, for any subdiagram of $\langle 12345678\rangle$, AzURITE will simply pinch propagators to obtain the graph, without running the backtracking algorithm again.

AzURITE also finds the discrete symmetries of a given graph. Propagatorsymmetry [index] provides the permutation symmetry of propagators. For example, with the inverse propagators given in eq. (B.3), the (physical) symmetry group of diagram $\langle 145678\rangle$ is given by PropagatorSymmetry $[\{1,4,5,6,7,8\}]$. The output is,

$\{\{z[1] \rightarrow z[1], z[4] \rightarrow z[4], z[5] \rightarrow z[5], z[6] \rightarrow z[6], z[7] \rightarrow z$ $[7], z[8] \rightarrow z[8]\},\{z[1] \rightarrow z[4], z[4] \rightarrow z[1], z[5] \rightarrow z[7], z[6]$ $\rightarrow z[6], z[7] \rightarrow z[5], z[8]->z[8]\}\}$

\footnotetext{
${ }^{8}$ Versions 10 and 11 of Mathematica have a problem in labelling edges of multi graphs: the multiple edges cannot be distinctly labelled. We expect that this issue will be solved in future versions of MATHEMATICA.
} 
where $z$ [i] denotes the Baikov variable $z_{i}$, namely, the $i$ th propagator.

On the other hand, the action of the symmetries on the momenta can be obtained by AzURITE's MomentaSymmetry[index]. For example, the output of MomentaSymmetry $[\{1,4,5,6,7,8\}]$ reads,

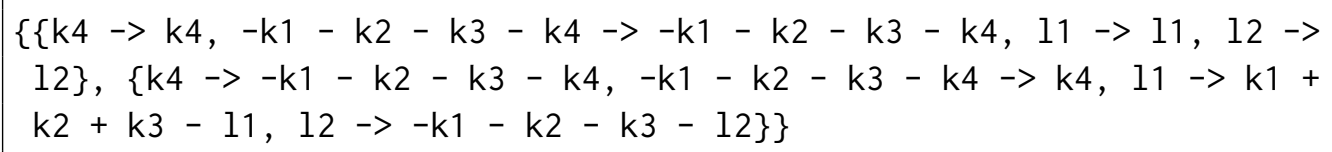

The non-trivial element is the affine transformation given in eq. (15). (Note that $-\mathrm{k} 1-\mathrm{k} 2-\mathrm{k} 3-\mathrm{k} 4$ means $\mathrm{k} 5$, since the latter is a linearly dependent momentum.)

\section{Appendix A.2.4. Master integrals}

DiagramAnalysis[index] provides the list of basis integrals for a given diagram, without considering its subdiagrams. It is useful for studying an individual diagram in detail. For example, with the inverse propagators given in eq. (B.3), DiagramAnalysis $[\{1,2,3,4,5,6,7,8\}]$ finds the master integrals of $\langle 12345678\rangle$. The output is,

$\{z[10], z[9], 1\}$

which means that there are three master integrals supported on the maximal cut $z_{1}=z_{2}=\ldots=z_{8}=0$. They are integrals with numerators $z_{10}=\left(l_{2}+k_{1}\right)^{2}$, $z_{9}=\left(l_{1}+k_{4}\right)^{2}$ and 1. Similarly, DiagramAnalysis $[\{1,4,5,6,7,8\}]$ gives \{\} , which means that this diagram has no master integrals which are supported on the maximal cut $z_{1}=z_{4}=z_{5}=z_{6}=z_{7}=z_{8}=0$. DiagramAnalysis has the following options,

- NumericMode with the default value True. This determines if the computation is carried out numerically.

- Characteristic with the default value 0 . This is the characteristic of the number field, which can be chosen as either a prime number $p$, or 0 . In the former case, the finite field $\mathbb{Z} / p \mathbb{Z}$ is used, while in the latter case the field of rational numbers $\mathbb{Q}$ is used.

- NumericD with the default value Null. When this value is a number, then the spacetime dimension will be set to this numerical value. Note that only rational non-integer values can be used. 
- WorkingPower with the default value 4 . This is the degree limit for the numerators appearing in the independent IBP identities, after Gaussian elimination. In general, to get the integral basis, we do not need to reduce all renormalizable terms by IBP identities.

- HighestPower with the default value 4. Occasionally, to get all IBP identities up to the degree specified by WorkingPower, we need IBP identities with the degrees higher than WorkingPower. Otherwise, the output basis may be redundant and contain integrals with the degree exactly the same as WorkingPower. HighestPower sets the limit for IBP identities in the intermediate steps. HighestPower should be greater than or than WorkingPower.

- Symmetry with the default value True. It determines if symmetries are used for the integral reduction.

- WatchingMode with the default value False. If it is set to be True, then the intermediate steps of the computations are printed.

DiagramAnalysis applies adaptive parametrizations. Hence if several external lines attach to one vertex, or if the diagram is factorizable, it will automatically determine a new list of ISPs. If the adaptive parametrization is used, the output may contain expressions mp2[...] which denotes Minkowski scalar products $(\ldots)^{2}$. Scalar products can be expressed as a function of the original propagators, via SPExpand[]. For example,

SPExpand[mp2[-k1 - k2 - k3 - k4 + 11]]

gives the output,

$-s 12-s 13-s 23+z[1]+z[4]-2 z[9]$

which means $\left(l_{1}+k_{5}\right)^{2}=-s_{12}-s_{13}-s_{23}+D_{1}+D_{4}-2 D_{9}$.

Note that DiagramAnalysis considers a diagram individually, and symmetries between different diagrams are ignored. For example, for the inverse propagators given in eq. (B.3), DiagramAnalysis determines both $\langle 158\rangle[1]$ and $\langle 478\rangle[1]$ as master integrals. However, they are equal by a discrete symmetry.

On the other hand, FindAllMIs[index] finds all master integrals within a diagram and all of its subdiagrams. It first finds the symmetries between different diagrams, and then determines the candidate diagrams for the search of 
master integrals. For example, all master integrals, including subdiagrams, for the inverse propagators given in eq. (B.3), can be found by calling FindAllMIs $[\{1,2,3,4,5,6,7,8\}]$. During the computation of FindAllMIs, the obtained master integrals are printed in the following format,

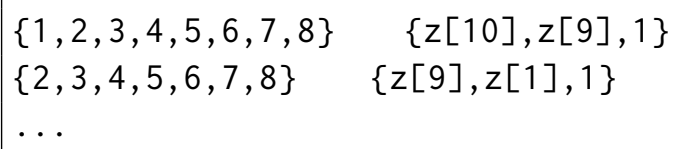

For each line the first entry is the list of propagators of a diagram, while the second entry is the list of numerators for master integrals of this topology. When the computation has finished, the total time used is also displayed. The output of FindAllMIs is a list which consists of items whose first element is the diagram index, and the second item is the list of numerators of master integrals.

After calling FindAllMIs, the associated diagrams of master integrals can be obtained and displayed by calling,

MIList $=$ FindAllMIs $[\{1,2,3,4,5,6,7,8\}]$;

FeynmanGraph[\#[[1]], DiagramExtendedOutput $\rightarrow$ True] \& /@ MIList

Most options of FindAllMIs are the same as those of DiagramAnalysis. However to speed up the search process, some default values are different:

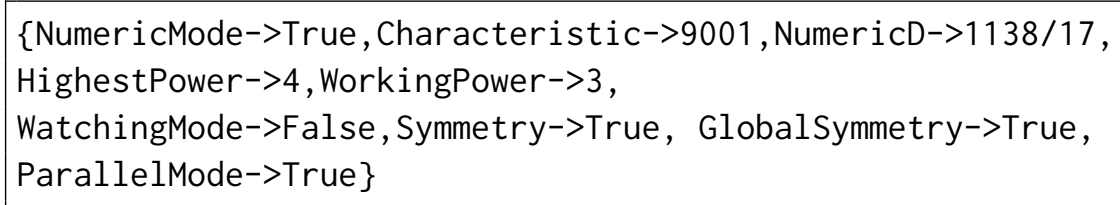

Here Globalsymmetry is a special option which determines whether the symmetries between different diagrams are used. ParallelMode is the option which indicates if the parallel computation is used. If its value is True, then the subdiagrams are assigned to several processors, and the integral basis searching can be significantly sped up.

\section{Appendix A.2.5. Analytic IBP identities evaluated on their maximal cut}

Analytic (or numerical) IBP identities evaluated on their maximal cut can be obtained by the IntegralRed command. For example, for the triple- 
box diagram (shown in Azurite code sample 1), the IBP identities on the maximal (10-propagator) cut can obtained via,

$\{$ MIs, IBP $\}=$ IntegralRed $[\{1,2,3,4,5,6,7,8,9,10\}]$;

where the variable MIs contains master integrals and IBP contains IBP identities evaluated on their maximal cut, in the form of replacement rules. The reduction of a specific integral can now be obtained,

Int $[1,1,1,1,1,1,1,1,1,1,-3,0,0,0,0] /$. IBP

where $\operatorname{Int}\left[a_{1}, \ldots, a_{k}, \ldots a_{n_{\mathrm{SP}}}\right]$ denotes the integral with the integrand,

$$
\frac{D_{k+1}^{-a_{k+1}} \cdots D_{n_{\mathrm{SP}}}^{-a_{n_{\mathrm{SP}}}}}{D_{1}^{a_{1}} \cdots D_{k}^{a_{k}}} .
$$

where $D_{k+1}, \ldots D_{n_{\mathrm{SP}}}$ are irreducible scalar products.

The options for IntegralRed are similar to those of DiagramAnalysis, except that the default values are for analytic computation:

$\{$ NumericMode- $>$ False, Characteristic $->0$, NumericD- $>$ Null, HighestPower $->4$, WorkingPower->4, WatchingMode->False, Symmetry->True\}

Note that IntegralRed does not use adaptive parametrization. Hence for IntegralRed, the kinematic input must correspond to a non-factorizable diagram whose external lines attach to distinct vertices.

\section{Appendix B. Integrals with squared propagators in Baikov repre- sentation on maximal cuts}

In this paper, we mainly discuss integrals without squared propagators. However, integrals with squared propagators do appear in various contexts, for example, importantly, in the context of differential equations [18, 19, 20, 21, 22, 23, 24. To explain the relation between integrals with and without squared propagators, in this section we derive the form of squared-propagator integrals in their Baikov representation on maximal cuts.

Recall that in eq. (19), the maximal-cut form of an integral without squared propagators is obtained by simply setting $z_{1}, \ldots, z_{m}$ to zero in the 
integrand. If a propagator $z_{j}$ is squared $(1 \leq j \leq m)$, then a residue computation is necessary to obtain the Baikov representation on the maximal cut. In the notation of eq. (19), the integral with the integrand $N /\left(D_{1} \cdots D_{j}^{2} \cdots D_{m}\right)$, evaluated on the maximal cut is proportional to,

$$
\begin{aligned}
\int \mathrm{d} z_{m+1} & \cdots \mathrm{d} z_{n_{\mathrm{SP}}^{\prime}} \oint_{\mathcal{C}_{j}} \mathrm{~d} z_{j} \frac{1}{z_{j}^{2}} F\left(0, \ldots, z_{j} \ldots, 0, z_{m+1}, \ldots, z_{n_{\mathrm{SP}}^{\prime}}\right)^{\frac{D-h}{2}} \\
& \times N\left(0, \ldots, z_{j}, \ldots, 0, z_{m+1}, \ldots, z_{n_{\mathrm{SP}}^{\prime}}\right)
\end{aligned}
$$

in Baikov representation. Here $\mathcal{C}_{j}$ is a small contour around the point $z_{j}=$ 0 . Furthermore, assume that the integrand has been reduced so that the numerator $N$ is independent of $z_{j}$. After evaluating this residue, the maximalcut form reads,

$$
\begin{gathered}
\int \mathrm{d} z_{m+1} \cdots \mathrm{d} z_{n_{\mathrm{SP}}^{\prime}} F\left(0, \ldots, 0, z_{m+1}, \ldots, z_{n_{\mathrm{SP}}^{\prime}}\right)^{\frac{D-h-2}{2}} \\
\times \frac{D-h}{2} \frac{\partial F}{\partial z_{j}}\left(0, \ldots, 0, z_{m+1}, \ldots, z_{n_{\mathrm{SP}}^{\prime}}\right) N\left(0, \ldots, 0, z_{m+1}, \ldots, z_{n_{\mathrm{SP}}^{\prime}}\right) .
\end{gathered}
$$

Therefore a squared-propagator integral evaluated on its maximal cut, in its Baikov representation, is equivalent to a $(D-2)$-dimensional integral without squared propagators. So by dimension-shift identities and IBP identities, a $D$-dimensional integral with squared propagators equals a linear combination of $D$-dimensional integrals without squared propagators.

As an example, consider the two-loop four-point massless double-box diagram with inverse propagators:

$$
\begin{array}{llll}
D_{1}=l_{1}^{2}, & D_{2}=\left(l_{1}-k_{1}\right)^{2}, & D_{3}=\left(l_{1}-K_{12}\right)^{2}, & D_{4}=\left(l_{1}+K_{12}\right)^{2}, \\
D_{5}=\left(l_{2}-k_{4}\right)^{2}, & D_{6}=l_{2}^{2}, & D_{7}=\left(l_{1}+l_{2}\right)^{2},
\end{array}
$$

where $k_{1}^{2}=k_{2}^{2}=k_{3}^{2}=k_{4}^{2}=0,\left(k_{1}+k_{2}\right)^{2}=s$ and $\left(k_{1}+k_{4}\right)^{2}=t$. As in eq. A.1., we define

$$
I\left[m_{1}, \ldots m_{9} ; D\right]=\int \frac{\mathrm{d}^{D} l_{1}}{i \pi^{D / 2}} \frac{\mathrm{d}^{D} l_{2}}{i \pi^{D / 2}} \frac{\left(l_{1}+k_{4}\right)^{-m_{8}}\left(l_{2}+k_{1}\right)^{-m_{9}}}{D_{1}^{m_{1}} \cdots D_{7}^{m_{7}}} .
$$

Define Baikov variables as, $z_{i} \equiv D_{i}, i=1, \ldots, 7, z_{8} \equiv\left(l_{1}+k_{4}\right)^{2}$ and $z_{9} \equiv$ $\left(l_{2}+k_{1}\right)^{2}$. The Baikov representation is,

$$
I\left[m_{1}, \ldots m_{9} ; D\right]=C(D) \int \prod_{i=1}^{9} \mathrm{~d} z_{i} F(z)^{\frac{D-6}{2}} \frac{z_{8}^{-m_{8}} z_{9}^{-m_{9}}}{z_{1}^{m_{1}} \ldots z_{7}^{m_{7}}},
$$


where $C(D)$ is a dimension-dependent prefactor. Now consider the maximal cut $z_{1}=\ldots=z_{7}=0$. For example,

$$
I[1,1,1,1,1,1,2,0,0 ; D]=\left.C(D) \int \mathrm{d} z_{8} \mathrm{~d} z_{9} \frac{D-6}{2}\left(\frac{\partial F}{\partial z_{7}} F(z)^{\frac{D-8}{2}}\right)\right|_{z_{1}=\ldots=z_{7}=0} .
$$

Hence on the maximal cut, $I[1,1,1,1,1,1,2,0,0 ; D]$ equals a $(D-2)$-dimensional integral with the numerator $\partial F / \partial z_{7}$, but without squared propagators. Using $(D-2)$-dimensional IBP identities, we obtain,

$$
I[1,1,1,1,1,1,2,0,0 ; D]=\frac{C(D)}{C(D-2)}\left(a_{1} B_{1}[D-2]+a_{2} B_{2}[D-2]\right),
$$

where

$$
\begin{aligned}
& B_{1}[D] \equiv I[1,1,1,1,1,1,1,0,0 ; D-2], \\
& B_{2}[D] \equiv I[1,1,1,1,1,1,1,-1,0 ; D-2],
\end{aligned}
$$

are the two master integrals of the double-box topology. The coefficients are $a_{1}=(D-6) s^{2} /(16(s+t))$ and $a_{2}=-(D-6) s(3 s+2 t) /(16 t(s+t))$. Here ... denotes integrals with fewer-than-seven propagators.

On the other hand,

$$
I\left[m_{1}, \ldots m_{9} ; D\right]=C(D) \int \prod_{i=1}^{9} \mathrm{~d} z_{i} F(z)^{\frac{D-8}{2}} \frac{z_{8}^{-m_{8}} z_{9}^{-m_{9}} F(z)}{z_{1}^{m_{1}} \ldots z_{7}^{m_{7}}} .
$$

which implies dimension-shift identities. Again using $(D-2)$-dimensional IBP identities,

$$
B_{i}[D]=\frac{C(D)}{C(D-2)}\left(T_{1 i} B_{1}[D-2]+T_{2 i} B_{2}[D-2]\right)+\ldots,
$$

where $i=1,2$. Now compare eqs. (B.7) and (B.10), and define

$$
\left(\begin{array}{l}
c_{1} \\
c_{2}
\end{array}\right) \equiv\left(\begin{array}{ll}
T_{11} & T_{12} \\
T_{21} & T_{22}
\end{array}\right)^{-1}\left(\begin{array}{l}
a_{1} \\
a_{2}
\end{array}\right)
$$

Then, on the maximal cut, the squared-propagator integral is related to integrals without squared propagators as $I[1,1,1,1,1,1,2,0,0 ; D]=c_{1} B_{1}[D]+$ $c_{2} B_{2}[D]+\ldots$ Here,

$$
c_{1}=-\frac{(D-5)(3 D-14)}{(D-6) t}, \quad c_{2}=-\frac{2(D-5)(D-4)}{(D-6) s t} .
$$


Note that the explicit form of $C(D)$ is not needed for deriving these coefficients.

This representation of integrals with squared propagators on maximal cuts clearly generalizes to integrals with squared propagators on non-maximal cuts.

\section{References}

[1] F. Tkachov, A Theorem on Analytical Calculability of Four Loop Renormalization Group Functions, Phys.Lett. B100 (1981) 65-68. doi : 10.1016/0370-2693(81)90288-4.

[2] K. Chetyrkin, F. Tkachov, Integration by Parts: The Algorithm to Calculate beta Functions in 4 Loops, Nucl.Phys. B192 (1981) 159-204. doi :10.1016/0550-3213(81)90199-1.

[3] A. V. Smirnov, A. V. Petukhov, The Number of Master Integrals is Finite, Lett. Math. Phys. 97 (2011) 37-44. arXiv:1004.4199, doi:10. 1007/s11005-010-0450-0.

[4] S. Laporta, High precision calculation of multiloop Feynman integrals by difference equations, Int.J.Mod.Phys. A15 (2000) 5087-5159. arXiv: hep-ph/0102033, doi:10.1016/S0217-751X(00)00215-7.

[5] S. Laporta, Calculation of master integrals by difference equations, Phys. Lett. B504 (2001) 188-194. arXiv:hep-ph/0102032, doi:10. 1016/S0370-2693(01)00256-8.

[6] C. Anastasiou, A. Lazopoulos, Automatic integral reduction for higher order perturbative calculations, JHEP 0407 (2004) 046. arXiv: hep-ph/ 0404258, doi :10.1088/1126-6708/2004/07/046.

[7] A. Smirnov, Algorithm FIRE - Feynman Integral REduction, JHEP 0810 (2008) 107. arXiv:0807.3243, doi:10.1088/1126-6708/2008/10/ 107.

[8] A. V. Smirnov, FIRE5: a C++ implementation of Feynman Integral REduction, Comput. Phys. Commun. 189 (2015) 182-191. arXiv:1408. 2372, doi:10.1016/j.cpc.2014.11.024. 
[9] C. Studerus, Reduze-Feynman Integral Reduction in C++, Comput.Phys.Commun. 181 (2010) 1293-1300. arXiv:0912.2546, doi: $10.1016 /$ j.cpc.2010.03.012.

[10] A. von Manteuffel, C. Studerus, Reduze 2 - Distributed Feynman Integral ReductionarXiv:1201.4330.

[11] R. N. Lee, Presenting LiteRed: a tool for the Loop InTEgrals REDuctionarXiv: 1212.2685 .

[12] A. von Manteuffel, R. M. Schabinger, A novel approach to integration by parts reduction, Phys. Lett. B744 (2015) 101-104. arXiv:1406.4513, doi:10.1016/j.physletb.2015.03.029.

[13] A. von Manteuffel, E. Panzer, R. M. Schabinger, On the Computation of Form Factors in Massless QCD with Finite Master Integrals, Phys. Rev. D93 (12) (2016) 125014. arXiv:1510.06758, doi:10.1103/PhysRevD. 93.125014.

[14] A. von Manteuffel, R. M. Schabinger, Quark and gluon form factors to four loop order in QCD: the $N_{f}^{3}$ contributions arXiv:1611.00795.

[15] T. Peraro, Scattering amplitudes over finite fields and multivariate functional reconstruction, JHEP 12 (2016) 030. $\operatorname{arXiv:1608.01902,}$ doi:10.1007/JHEP12(2016)030.

[16] J. Gluza, K. Kajda, D. A. Kosower, Towards a Basis for Planar TwoLoop Integrals, Phys.Rev. D83 (2011) 045012. arXiv:1009.0472, doi: 10.1103/PhysRevD.83.045012.

[17] R. M. Schabinger, A New Algorithm For The Generation Of UnitarityCompatible Integration By Parts Relations, JHEP 1201 (2012) 077. arXiv:1111.4220, doi:10.1007/JHEP01(2012)077.

[18] A. V. Kotikov, Differential equations method: New technique for massive Feynman diagrams calculation, Phys. Lett. B254 (1991) 158-164. doi: 10.1016/0370-2693(91)90413-K.

[19] A. V. Kotikov, Differential equation method: The Calculation of N point Feynman diagrams, Phys. Lett. B267 (1991) 123-127, [Erratum: Phys. Lett.B295,409(1992)]. doi:10.1016/0370-2693(91)90536-Y,10.1016/ 0370-2693(92)91582-T. 
[20] Z. Bern, L. J. Dixon, D. A. Kosower, Dimensionally regulated pentagon integrals, Nucl. Phys. B412 (1994) 751-816. arXiv:hep-ph/9306240, doi : 10.1016/0550-3213(94)90398-0.

[21] E. Remiddi, Differential equations for Feynman graph amplitudes, Nuovo Cim. A110 (1997) 1435-1452. arXiv: hep-th/9711188.

[22] T. Gehrmann, E. Remiddi, Differential equations for two loop four point functions, Nucl.Phys. B580 (2000) 485-518. arXiv:hep-ph/9912329, doi:10.1016/S0550-3213(00)00223-6.

[23] J. Ablinger, A. Behring, J. Blmlein, A. De Freitas, A. von Manteuffel, C. Schneider, Calculating Three Loop Ladder and V-Topologies for Massive Operator Matrix Elements by Computer Algebra, Comput. Phys. Commun. 202 (2016) 33-112. arXiv:1509.08324, doi: $10.1016 /$ j.cpc.2016.01.002.

[24] J. M. Henn, Multiloop integrals in dimensional regularization made simple, Phys. Rev. Lett. 110 (2013) 251601. arXiv:1304.1806, doi: 10.1103/PhysRevLett.110.251601.

[25] R. N. Lee, Reducing differential equations for multiloop master integrals, JHEP 04 (2015) 108. arXiv:1411.0911, doi:10.1007/JHEP04(2015) 108.

[26] C. Meyer, Transforming differential equations of multi-loop Feynman integrals into canonical formarXiv:1611.01087.

[27] E. Remiddi, L. Tancredi, Differential equations and dispersion relations for Feynman amplitudes. The two-loop massive sunrise and the kite integral, Nucl. Phys. B907 (2016) 400-444. arXiv:1602.01481, doi: $10.1016 / j$. nuclphysb.2016.04.013.

[28] R. Bonciani, V. Del Duca, H. Frellesvig, J. M. Henn, F. Moriello, V. A. Smirnov, Two-loop planar master integrals for Higgs $\rightarrow 3$ partons with full heavy-quark mass dependencearXiv:1609.06685.

[29] A. Primo, L. Tancredi, On the maximal cut of Feynman integrals and the solution of their differential equationsarXiv:1610.08397. 
[30] K. J. Larsen, Y. Zhang, Integration-by-parts reductions from unitarity cuts and algebraic geometry, Phys. Rev. D93 (4) (2016) 041701. arXiv: 1511.01071, doi:10.1103/PhysRevD.93.041701.

[31] H. Ita, Two-loop Integrand Decomposition into Master Integrals and Surface Terms, Phys. Rev. D94 (11) (2016) 116015. arXiv:1510.05626, doi:10.1103/PhysRevD.94.116015.

[32] W. Decker, G.-M. Greuel, G. Pfister, H. Schönemann, Singular 4-02 - A computer algebra system for polynomial computations, http: //www. singular.uni-kl.de (2015).

[33] R. N. Lee, A. A. Pomeransky, Critical points and number of master integrals, JHEP 11 (2013) 165. arXiv:1308.6676, doi:10.1007/ JHEP11(2013)165.

[34] G. Ossola, C. G. Papadopoulos, R. Pittau, Reducing full one-loop amplitudes to scalar integrals at the integrand level, Nucl.Phys. B763 (2007) 147-169. arXiv: hep-ph/0609007, doi:10.1016/j.nuclphysb.2006.11. 012.

[35] G. Ossola, C. G. Papadopoulos, R. Pittau, CutTools: A Program implementing the OPP reduction method to compute one-loop amplitudes, JHEP 0803 (2008) 042. arXiv:0711.3596, doi:10.1088/1126-6708/ 2008/03/042.

[36] R. Ellis, Z. Kunszt, K. Melnikov, G. Zanderighi, One-loop calculations in quantum field theory: from Feynman diagrams to unitarity cutsarXiv: 1105.4319 .

[37] R. Ellis, W. Giele, Z. Kunszt, A Numerical Unitarity Formalism for Evaluating One-Loop Amplitudes, JHEP 0803 (2008) 003. arXiv: 0708. 2398, doi:10.1088/1126-6708/2008/03/003.

[38] P. Mastrolia, G. Ossola, On the Integrand-Reduction Method for TwoLoop Scattering Amplitudes, JHEP 1111 (2011) 014. arXiv:1107.6041, doi : 10.1007/JHEP11(2011)014.

[39] S. Badger, H. Frellesvig, Y. Zhang, Hepta-Cuts of Two-Loop Scattering Amplitudes, JHEP 1204 (2012) 055. arXiv:1202.2019, doi:10.1007/ JHEP04(2012) 055. 
[40] Y. Zhang, Integrand-Level Reduction of Loop Amplitudes by Computational Algebraic Geometry Methods, JHEP 1209 (2012) 042. arXiv: 1205.5707, doi : 10.1007/JHEP09 (2012) 042.

[41] P. Mastrolia, E. Mirabella, G. Ossola, T. Peraro, Scattering Amplitudes from Multivariate Polynomial Division, Phys.Lett. B718 (2012) 173-177. arXiv:1205.7087, doi:10.1016/j.physletb.2012.09.053.

[42] P. A. Baikov, Explicit solutions of the three loop vacuum integral recurrence relations, Phys. Lett. B385 (1996) 404-410. arXiv:hep-ph/ 9603267, doi:10.1016/0370-2693(96)00835-0.

[43] W. L. van Neerven, J. A. M. Vermaseren, LARGE LOOP INTEGRALS, Phys. Lett. B137 (1984) 241-244. doi:10.1016/0370-2693(84) 90237-5.

[44] R. N. Lee, Calculating multiloop integrals using dimensional recurrence relation and D-analyticity, Nucl. Phys. Proc. Suppl. 205-206 (2010) 135140. arXiv:1007.2256, doi:10.1016/j.nuclphysbps.2010.08.032.

[45] B. Bollobás, Modern graph theory, Vol. 184 of Graduate Texts in Mathematics, Springer-Verlag, New York, 1998. doi:10.1007/ 978-1-4612-0619-4. URL http://dx.doi.org/10.1007/978-1-4612-0619-4

[46] P. Mastrolia, T. Peraro, A. Primo, Adaptive Integrand Decomposition in parallel and orthogonal space, JHEP 08 (2016) 164. arXiv:1605.03157, doi : 10.1007/JHEP08(2016)164.

[47] R. N. Lee, Modern techniques of multiloop calculations, in: Proceedings, 49th Rencontres de Moriond on QCD and High Energy Interactions: La Thuile, Italy, March 22-29, 2014, 2014, pp. 297-300. arXiv:1405.5616. URL https://inspirehep.net/record/1297497/files/arXiv:1405. 5616.pdf

[48] D. A. Kosower, K. J. Larsen, Maximal Unitarity at Two Loops, Phys. Rev. D85 (2012) 045017. arXiv:1108.1180, doi:10.1103/PhysRevD. 85.045017 .

[49] H. Johansson, D. A. Kosower, K. J. Larsen, An Overview of Maximal Unitarity at Two Loops[PoSLL2012,066(2012)]. arXiv:1212.2132. 
[50] S. Caron-Huot, K. J. Larsen, Uniqueness of two-loop master contours, JHEP 1210 (2012) 026. arXiv:1205.0801, doi:10.1007/JHEP10(2012) 026.

[51] H. Johansson, D. A. Kosower, K. J. Larsen, Two-Loop Maximal Unitarity with External Masses, Phys.Rev. D87 (2013) 025030. arXiv: 1208.1754, doi:10.1103/PhysRevD.87.025030.

[52] H. Johansson, D. A. Kosower, K. J. Larsen, Maximal Unitarity for the Four-Mass Double BoxarXiv:1308.4632.

[53] M. Sogaard, Y. Zhang, Multivariate Residues and Maximal Unitarity, JHEP 12 (2013) 008. arXiv:1310.6006, doi:10.1007/JHEP12(2013) 008 .

[54] H. Hauser, G. Müller, Affine varieties and lie algebras of vector fields, manuscripta mathematica 80 (1) (1993) 309-337. doi:10.1007/ BF03026556.

URL http://dx.doi.org/10.1007/BF03026556

[55] D. A. Cox, J. B. Little, D. O'Shea, Using algebraic geometry, Graduate texts in mathematics, Springer, New York, 1998. URL http://opac. inria. fr/record=b1094391

[56] S. Di Vita, P. Mastrolia, U. Schubert, V. Yundin, Three-loop master integrals for ladder-box diagrams with one massive leg, JHEP 09 (2014) 148. arXiv:1408.3107, doi:10.1007/JHEP09(2014)148.

[57] J. C. Faugère, A new efficient algorithm for computing gröbner bases without reduction to zero (f5), in: Proceedings of the 2002 International Symposium on Symbolic and Algebraic Computation, ISSAC '02, ACM, New York, NY, USA, 2002, pp. 75-83. doi:10.1145/780506.780516.

URL http://doi .acm.org/10.1145/780506.780516 